\title{
Genetic variation in the hepatic lipase gene and the risk of coronary heart disease among US diabetic men: potential interaction with obesity
}

Received: 18 November 2005 / Accepted: 20 February 2006 / Published online: 29 March 2006

(C) Springer-Verlag 2006

\begin{abstract}
Aims/hypothesis: The $-514 \mathrm{C}$ to $\mathrm{T}$ polymorphism of the hepatic lipase gene (LIPC) has been associated with lowered LIPC activity and elevated HDL-cholesterol concentrations. Previous findings on the association of this polymorphism with the risk of CHD are inconsistent. Moreover, data on this association among diabetic patients are limited. We investigated the association of the LIPC polymorphism with CHD risk among US diabetic men and evaluated whether this association was modified by adiposity status. Subjects and methods: The case group consisted of 220 diabetic men who were recruited from the Health Professionals Follow-up Study (years 1986-2000) and were free of cardiovascular disease at baseline, but subsequently developed CHD. A total of 641 diabetic men from the same study but without cardiovascular disease constituted the control group. Results: No overall association between the LIPC polymorphism and CHD risk was observed. However, we did observe a significant interaction between this polymorphism and BMI in association with CHD risk. Among obese men, after adjustment for age, duration of diabetes and major lifestyle factors, the CT or TT genotype was associated with an increased CHD risk compared with the $\mathrm{CC}$ genotype (odds ratio [OR]
\end{abstract}

C. Zhang $(\bowtie) \cdot$ R. Lopez-Ridaura $\cdot$ E. B. Rimm ·

T. Li - D. J. Hunter · F. B. Hu

Department of Nutrition,

Harvard School of Public Health,

665 Huntington Avenue,

Boston, MA 02115, USA

e-mail: nhcui@channing.harvard.edu

Tel.: +1-617-4324973

Fax: +1-617-4322435

R. Lopez-Ridaura · E. B. Rimm · D. J. Hunter · F. B. Hu Channing Laboratory, Department of Medicine,

Brigham and Women's Hospital and Harvard Medical School, Boston, MA, USA

E. B. Rimm · D. J. Hunter · F. B. Hu

Department of Epidemiology,

Harvard School of Public Health,

Boston, MA, USA
2.52, 95\% CI 1.08-5.90); the corresponding ORs $(95 \%$ CI $)$ were $0.99(0.58,1.69)$ for overweight men $\left(25 \leq \mathrm{BMI}<30 \mathrm{~kg} / \mathrm{m}^{2}\right)$ and $0.37(0.17,0.79)$ for lean men $\left(B M I<25 \mathrm{~kg} / \mathrm{m}^{2}\right)$ ( $p$ for interaction 0.001). Stratified analyses by waist circumference (tertiles) showed a similar pattern of interaction (adjusted $p$ for interaction 0.023). Conclusion/interpretation: These data suggest that obesity may modify the association between the LIPC C $(-514)$ $\mathrm{T}$ polymorphism and CHD risk among diabetic men.

Keywords Coronary heart disease $\cdot$ Diabetes $\cdot$ Gene Hepatic lipase · Interaction · Obesity

Abbreviations CABG: coronary artery bypass grafting HPFS: Health Professionals Follow-up Study - IDL: intermediate density lipoprotein - LIPC: hepatic lipase LIPC: hepatic lipase gene $\cdot$ OR: odds ratio

\section{Introduction}

A decreased plasma HDL-cholesterol (HDL-C) concentration is an established risk factor for CHD among diabetic patients [1]. Allelic variations at the gene encoding hepatic lipase (LIPC) account for up to $25 \%$ of the variability in plasma HDL-C concentrations [2]. LIPC is a lipolytic enzyme that is primarily synthesised in the parenchymal liver cells and secreted and bound extracellularly to the endothelial cells in the hepatic sinusoids. It plays a crucial role in remodelling remnant lipoprotein, LDL and HDL [3-5] as both a catalyst and a ligand. Specifically, it catalyses the conversion of large HDL2 particles to small, dense HDL and acts on large LDL and intermediate density lipoprotein (IDL) particles to form small, dense LDL (sdLDL) particles. In addition, it can facilitate the hepatic uptake of lipoproteins, including HDL, by acting as a ligand mediating the binding and uptake of lipoproteins through proteoglycans and/or receptor pathways $[6,7]$.

LIPC activity has been reported to be determined by sex $[8,9]$, body adiposity [10], dietary fat intake [11, 12], 
alcohol consumption [13, 14], physical activity [15-17] and, most importantly, by genotype. The human hepatic lipase gene (LIPC) maps to chromosome 15q21 [18]. Four polymorphisms in the $5^{\prime}$-flanking region of this gene $(\mathrm{G} \rightarrow \mathrm{A}$ at position $-250, \mathrm{C} \rightarrow \mathrm{T}$ at $-514, \mathrm{~T} \rightarrow \mathrm{C}$ at -710 and $\mathrm{A} \rightarrow \mathrm{G}$ at -763$)$ are in complete linkage disequilibrium [19]. They were designated as the $-514 \mathrm{C}$ and $\mathrm{T}$ alleles together $[4,19,20]$. The $\mathrm{C} \rightarrow \mathrm{T}$ substitution disrupts the upstream stimulatory factor 1 binding site present in the proximal promoter region of the hepatic lipase gene and has been shown to decrease transcriptional activity of the LIPC promoter in vitro [21, 22]. Furthermore, it accounted for up to $38 \%$ of the variation in LIPC activity [23] and was associated with increased concentrations of plasma HDL and HDL2 and more buoyant LDL particles [6, 20, 24, 25].

The relationship of the LIPC polymorphism to CHD risk has not been clearly established. Findings from several studies suggested a pro-atherogenic role of this polymorphism [24, 26-31]. The LIPC $\mathrm{C}(-514) \mathrm{T}$ polymorphism was associated with reduced coronary flow reserve (an early marker of atherosclerosis) [28], coronary artery calcification [26] and increased risk of CHD [27, 29-31]. However, other studies reported no association or an inverse association with CHD risk [20, 32-34]. We speculated that some of the inconsistencies may be, at least in part, due to different study population characteristics, such as underlying environmental factors that can affect lipid metabolism. Indeed, in our previous study, we observed that body adiposity significantly modified the association between the LIPC polymorphism and plasma HDL concentrations [35]; only among lean diabetic men was the LIPC polymorphism associated with higher HDL-C concentrations, whereas obesity abolished the beneficial effect of the polymorphism on HDL-C concentrations. In the present study we investigated the relationship between the LIPC -514 polymorphism and the risk of CHD and evaluated whether this association was modified by adiposity status among diabetic patients.

\section{Subjects and methods}

\section{Study background}

The Health Professionals Follow-up Study (HPFS) is a prospective cohort study of 51,529 American male health professionals who were aged $40-75$ years at study initiation in 1986. This cohort has been and continues to be followed through biennial mailed questionnaires focusing on various lifestyle factors and health outcomes. In addition, between 1993 and 1999 (more than 95\% of them between 1993 and 1995), 18,159 study participants provided blood samples by overnight courier. Among them, 999 (>97\% were people of European extraction) had confirmed type 2 diabetes (at baseline or during follow up, 1986-2000). From this group of diabetic men, we excluded those with reported CHD or stroke at baseline, those who developed stroke or other non-coronary cardiovascular disease during follow-up, and those who developed CHD before diabetes was diagnosed (total exclusions, 138). Among the remaining 861 diabetic men, 220 who developed CHD (i.e. non-fatal myocardial infarction, $n=66$; fatal CHD, $n=18$; coronary artery bypass grafting [CABG], $n=136$ ) constituted the case group and 641 diabetic men who remained free of CHD and stroke constituted the control group. The 201 cases and 604 control subjects for whom complete data on both LIPC genotype and BMI existed constituted the analytical population of this study. The participants provided written informed consent to be included in the study and all investigations were approved by the institutional review board ethics committee.

\section{Diagnosis of type 2 diabetes}

A diagnosis of diabetes was established when at least one of the following criteria was reported on a supplementary questionnaire sent to all men who reported a diagnosis of diabetes in any biennial follow-up questionnaire: (1) one or more classic symptoms (excessive thirst, polyuria, weight loss, hunger or coma) plus a fasting plasma glucose concentration $\geq 7.8 \mathrm{mmol} / 1$ or a random plasma glucose concentration $\geq 11.1 \mathrm{mmol} / \mathrm{l}$; (2) at least two elevated plasma glucose concentrations on different occasions (fasting $\geq 7.8 \mathrm{mmol} / 1$ and/or random $\geq 11.1 \mathrm{mmol} / 1$ and/or $\geq 11.1 \mathrm{mmol} / \mathrm{l}$ after $2 \mathrm{~h}$ or more on oral glucose tolerance testing) in the absence of symptoms; or (3) treatment with hypoglycaemic medication (insulin or oral hypoglycaemic agents). We used the National Diabetes Data Group criteria [36] to define diabetes because the majority of our cases were diagnosed before the release of the American Diabetes Association criteria [37]. Men with type 1 diabetes were excluded. A validation study in a subsample of the HPFS demonstrated that our supplementary questionnaire is highly reliable in confirming diabetes diagnosis [38].

Diagnosis of cardiovascular end-points

The cardiovascular end-points for this analysis comprised fatal CHD, non-fatal myocardial infarction, $\mathrm{CABG}$ and percutaneous transluminal coronary angioplasty occurring between the return of the 1986 questionnaire and 31 January 2000. The end-points did not include angina pectoris. Non-fatal myocardial infarction was confirmed by a review of medical records based on World Health Organization criteria that included characteristic symptoms with either typical electrocardiographic changes or elevations of cardiac enzymes [39]. Probable cases of myocardial infarction (no available records but confirmed by hospitalisation and information from telephone interview/letter) were also included in the analysis. Confirmation of CABG or percutaneous transluminal coronary angioplasty was based on self-report only; hospital records obtained for a sample of 102 men in the HPFS confirmed the procedure for $96 \%$ [40]. Deaths were reported by next of kin, the postal system, and through records of the National Death 
Index. Using all sources combined, it was estimated that follow-up for deaths was $>98 \%$ complete [41]. Fatal CHD was confirmed by reviewing medical records or autopsy reports with the permission of the next of kin. Physicians who reviewed the records had no knowledge of the selfreported risk factor status. Sudden deaths (deaths within $1 \mathrm{~h}$ of symptom onset in men without known disease that could explain death) were included in the fatal CHD category.

Measurements of body weight and waist circumference and ascertainment of characteristics of participants

At baseline, participants were asked to report their height (in inches; 1 in=2.54 cm) and current body weight (in pounds; $1 \mathrm{lb}=0.45 \mathrm{~kg}$ ); weight was then updated during the biennial follow-up. In 1987, HPFS participants were asked in a supplementary questionnaire to measure their waist circumference with a paper tape and were given detailed measuring directions. BMI was calculated as weight in kilograms divided by height squared in metres. Waist circumference was used as an indicator of central obesity. Self-reports of body weight, waist circumference and height have been shown to be highly correlated with technician-measured weight, waist circumference and height $(r=0.97,0.95$ and 0.94 , respectively) in HPFS participants [42].

Participants also provided information biennially on their cigarette smoking, aspirin use and physical activity. Physical activity (metabolic equivalent task [MET] hours/ week) was calculated from the reported time spent on various activities, weighting each activity by its intensity level. History of high blood pressure was determined from self-reports preceding the blood collection. Family history of CHD was reported in 1986. Alcohol intake was estimated with a dietary questionnaire in 1986.

\section{Laboratory analysis methods}

Details about the blood collection and processing procedure have been reported previously [43]. Briefly, approximately $95 \%$ of participants in the present study provided blood samples in 1993 and 1994. Blood samples were collected in three 10-ml liquid EDTA blood tubes, placed on ice packs stored in Styrofoam containers and returned to our laboratory by overnight courier; over $95 \%$ of the samples arrived within $24 \mathrm{~h}$. After receipt, the chilled blood was centrifuged, divided into plasma, erythrocytes and buffy coat, and stored in continuously monitored nitrogen freezers at temperatures not higher than $-130^{\circ} \mathrm{C}$. We requested information on the date and time of drawing the blood sample and the time elapsed since the preceding meal to identify non-fasting $(<8 \mathrm{~h})$ subjects. Measurements of lipid profiles were done among diabetic men who developed CHD after their blood samples were collected in 1993-1994 $(n=109)$ and their controls $(n=604)$. All lipid profiles were assayed in the laboratory of N. Rifai
(The Children's Hospital, Boston, MA, USA), which was certified by the NHLBI/CDC (National Heart, Lung and Blood Institute/Centers for Disease Control) Lipid Standardization Program. All assays except the ELISA and RIA employed a Hitachi 911 analyser (Roche Diagnostics, Indianapolis, IN, USA). Concentrations of total cholesterol, triacylglycerols and HDL-C were analysed simultaneously with enzymatic assays with CVs of $1.7,1.8$ and $2.5 \%$, respectively. LDL-cholesterol (LDL-C) was determined by a homogeneous direct method (Genzyme, Cambridge, MA, USA), with $\mathrm{CV}<3.1 \%$. Measurement of $\mathrm{HbA}_{1 \mathrm{c}}$ was based on turbidimetric immunoinhibition using haemolysed packed red cells. The intra-assay $\mathrm{CVs}$ at $\mathrm{HbA}_{1 \mathrm{c}}$ values of 5.5 and 9.1 were 1.9 and $3.0 \%$, respectively.

DNA was extracted from the buffy coat fraction of centrifuged blood using the QIAmp Blood Kit (Qiagen, Chatsworth, CA, USA). The genotyping technique was Taqman SNP allelic discrimination by means of an ABI 7900HT Sequence Detection System (Applied Biosystems, Foster City, CA, USA). Primers and probes are available on request.

\section{Statistical analysis}

Frequency distributions of characteristics of study subjects were examined according to case-control status. Student's $t$ tests and $\chi^{2}$ tests were used for comparisons of means and proportions. Because of the small number of subjects homozygous for the less common allele (genotype TT; nine cases, 25 controls) and similar magnitude of associations with CHD risk for individuals with this genotype and heterozygotes (genotype CT), individuals with genotypes $\mathrm{CT}$ and TT were grouped together.

Unconditional logistic regression was used to calculate odds ratios (OR) and 95\% CIs for the association between the LIPC genotype and CHD risk adjusted for risk factors for CHD. Adjustment for baseline variables (age, smoking status [never, past, current smoker], physical activity [quartile], alcohol consumption [non-drinker, $<5.0,<10.0$, $>10.0 \mathrm{~g} /$ day], total energy intake), and duration of diabetes changed the OR slightly, but we kept these variables in the final model because of their recognition as risk factors for CHD and interpretable variables that may account for the heterogeneity of study participants. Adjustment for other covariates, such as family history of myocardial infarction, aspirin use, history of hypertension and hypercholesterolaemia at baseline, did not show a significant effect on the ORs, and because they might be intermediates for the association they were not included in the final models.

Stratified analyses were conducted to examine whether the association between the LIPC polymorphism and CHD risk was modified by overall body adiposity status (lean, BMI <25; overweight, BMI 25.0-29.9; obese, BMI $\geq 30 \mathrm{~kg} /$ $\mathrm{m}^{2}$ ) defined using the World Health Organization criteria [44], and central obesity status (waist circumference: tertile [high, medium, low]) at baseline. Interactions between LIPC genotypes and BMI and waist circumference were 
assessed using a cross-product term between genotypes and these factors. Tests for interactions were performed using likelihood ratio tests by comparing two nested multivariate models with and without the interaction term. All reported $p$ values are two-tailed and statistical significance was defined at the $\alpha=0.05$ level. All analyses were performed with SAS version 8.12 software (SAS Institute, Cary, NC, USA).

\section{Results}

Compared with those without CHD, diabetic men who developed CHD tended to be older and were more likely to have a family history of myocardial infarction and to have hypertension and hypercholesterolaemia at baseline (Table 1). They were also more likely to use aspirin at baseline, probably because of their elevated cardiovascular risk factors. Plasma concentrations of LDL were higher and HDL-C lower among cases than controls.

Genotype frequencies did not deviate from HardyWeinberg equilibrium either among cases or among controls ( $p=0.97$ and $p=0.92$, respectively). Overall, genotype distributions of the LIPC C $(-514) \mathrm{T}$ polymorphism were not statistically significantly different between cases and controls ( $p=0.87, \chi^{2}$ test) (Table 2$)$. The frequency of the $-514 \mathrm{~T}$ allele was $21 \%$ for cases and $20 \%$ for controls. Thirty-three percent of both cases and controls were heterozygous for the $\mathrm{T}$ allele and $4 \%$ of both cases and controls were homozygous for the $\mathrm{T}$ allele. Because of the small number of subjects homozygous for the less common allele, individuals with CT and TT genotypes were grouped together in the following analyses. There was no evidence of a statistically significant association between the LIPC polymorphism (CT/TT genotype) and CHD risk before and after adjustment for age, BMI, smoking status, alcohol consumption, physical activity, duration of diabetes, and total energy intake (OR [95\% CI]: age-adjusted, 1.07 [0.76, 1.50]; multivariate-adjusted, 0.96 [0.67, 1.38]). Additional adjustment for family history of myocardial infarction, history of hypertension and hypercholesterolaemia, and use of aspirin at baseline did not change the results materially (adjusted OR [95\% CI]: 0.94 [0.66, 1.35]). In addition, results did not change appreciably after we excluded subjects taking cholesterol-lowering medications at baseline $(n=14)$. We therefore included these subjects in subsequent analyses.

The association between the LIPC -514 polymorphism and risk of CHD varied significantly according to BMI status (Table 3). After adjustment for smoking status, alcohol consumption, physical activity, duration of diabetes and total energy intake, among lean men $\left(\mathrm{BMI}<25 \mathrm{~kg} / \mathrm{m}^{2}\right.$ ), those with the CT or TT genotype had a $64 \%$ lower risk of CHD (age-adjusted OR $=0.36$, $95 \%$ CI $0.17,0.79)$ than those with the CC genotype. No significant association was observed among overweight men $\left(25 \leq \mathrm{BMI}<30 \mathrm{~kg} / \mathrm{m}^{2}\right)$. The CT or TT genotype, however, was associated with more than twofold greater risk of CHD among obese men (multivariateadjusted $\mathrm{OR}=2.52,95 \%$ CI 1.08, 5.90). The interaction between the LIPC C $(-514) \mathrm{T}$ polymorphism and BMI in relation to CHD risk (likelihood, $\chi^{2}=12.31, \mathrm{df}=1, p$ for interaction $=0.001$ ) was statistically significant. The results did not change after excluding those who reported taking

Table 1 Comparison of cardiovascular risk factors ${ }^{\mathrm{a}}$ between CHD cases and controls at baseline (1986) among US diabetic men

\begin{tabular}{|c|c|c|c|}
\hline Characteristic & $\begin{array}{l}\text { CHD cases } \\
n=201\end{array}$ & $\begin{array}{l}\text { Controls } \\
n=604\end{array}$ & $p$ value \\
\hline Age (years) & $59.2 \pm 7.4$ & $55.0 \pm 8.6$ & $<0.001$ \\
\hline BMI $\left(\mathrm{kg} / \mathrm{m}^{2}\right)$ & $28.0 \pm 4.6$ & $28.0 \pm 4.6$ & 0.32 \\
\hline Waist circumference $(\mathrm{cm})$ & $102.4 \pm 13.2$ & $101.3 \pm 11.4$ & 0.35 \\
\hline Family history of MI (\%) & 20.9 & 12.1 & 0.002 \\
\hline History of hypertension (\%) & 48.3 & 31.5 & $<0.001$ \\
\hline History of hypercholesterolaemia (\%) & 25.9 & 12.1 & $<0.001$ \\
\hline Physical activity (MET-hours/week) & $14.4 \pm 19.3$ & $14.2 \pm 17.8$ & 0.86 \\
\hline Total energy intake (kcal/day) & $2,024 \pm 618$ & $2,020 \pm 635$ & 0.94 \\
\hline Alcohol consumption (g/day) & $8.9 \pm 15.6$ & $11.0 \pm 16.5$ & 0.10 \\
\hline Never smokers $(\%)$ & 35.8 & 39.4 & 0.70 \\
\hline Aspirin users $(\%)$ & 45.3 & 29.5 & $<0.001$ \\
\hline \multicolumn{4}{|l|}{ Plasma lipoprotein ${ }^{\mathrm{a}}(\mathrm{mmol} / \mathrm{l})$} \\
\hline Triglyceride $^{\mathrm{b}}$ & $2.2 \pm 1.1$ & $2.0 \pm 1.1$ & 0.12 \\
\hline Cholesterol & $5.6 \pm 1.0$ & $5.5 \pm 1.1$ & 0.07 \\
\hline HDL-C & $1.0 \pm 0.2$ & $1.1 \pm 0.3$ & 0.002 \\
\hline LDL-C & $3.4 \pm 0.9$ & $3.2 \pm 0.9$ & 0.02 \\
\hline
\end{tabular}

All data are mean \pm SD unless otherwise indicated

MET metabolic equivalent task, $M I$ myocardial infarction

${ }^{a}$ Only for subjects whose blood sample was available before CHD was identified (109 CHD cases, 604 controls)

${ }^{\mathrm{b}}$ Only for subjects whose blood sample was available before CHD was identified and who fasted before blood was drawn (60 CHD cases, 335 controls) 
Table 2 Association between LIPC genotypes and the risk of CHD among US diabetic men

\begin{tabular}{lccll}
\hline LIPC genotype & Cases (201) & Controls (604) & $\begin{array}{l}\text { Age-adjusted }^{\mathrm{a}} \\
\text { OR (95\% CI) }\end{array}$ & $\begin{array}{l}\text { Multivariate-adjusted }^{\mathrm{b}} \\
\text { OR (95\% CI) }\end{array}$ \\
\hline $\mathrm{CC}$ & 126 & 382 & 1.00 referent & 1.00 referent \\
CC/TT & 75 & 222 & $1.07(0.76,1.50)$ & $0.96(0.67,1.38)$ \\
$\mathrm{CT}$ & 66 & 197 & $1.06(0.74,1.50)$ & $0.93(0.64,1.35)$ \\
TT & 9 & 25 & $1.17(0.52,2.63)$ & $1.21(0.52,2.83)$ \\
\hline
\end{tabular}

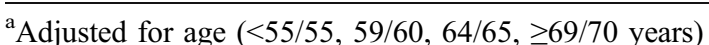

${ }^{\mathrm{b}}$ Adjusted for age, duration of diabetes, and the following lifestyle factors: alcohol consumption (non-drinker, $<5.0,<10.0,>10.0 \mathrm{~g} / \mathrm{day}$ ), smoking (never, past, current), physical activity (quartile), and total energy (kcal/day)

cholesterol-lowering medications. The interaction was attenuated but remained statistically significant after adjustment for HDL-C concentration ( $p$ for interaction=0.01). Further adjustment for LDL-C, $\mathrm{HbA}_{1 \mathrm{c}}$, family history of myocardial infarction, history of hypertension and hypercholesterolaemia, and use of aspirin did not appreciably alter the results ( $p$ for interaction $=0.002$ ).

Similarly, we observed a significant interaction between the $L I P C-514$ polymorphism and waist circumference (multivariate-adjusted $p$ for interaction $=0.023$ ) in association with CHD risk. The LIPC -514 polymorphism was related to an elevation of CHD risk confined to diabetic men with greater waist circumference. Among 217 men whose waist circumference was equal to or greater than $105.2 \mathrm{~cm}$ (the highest tertile of the study participants), the CT or TT genotype was associated with approximately two-fold increased risk of CHD (multivariate-adjusted $\mathrm{OR}=1.99,95 \%$ CI $0.98,4.10)$. Corresponding ORs were 0.86 for men with waist circumference in the middle tertile and 0.57 for those in the lowest tertile.

Considering that altered LIPC activity has been related to lifestyle factors, including dietary fat intake, physical activity, and alcohol consumption, we further examined whether these factors modulated the association between the LIPC -514 polymorphism and CHD risk. No statistically significant interactions between these factors

Table 3 Association between $L I P C$ genotypes and the risk of CHD according to BMI and waist circumference ${ }^{\mathrm{a}}$ among US diabetic men

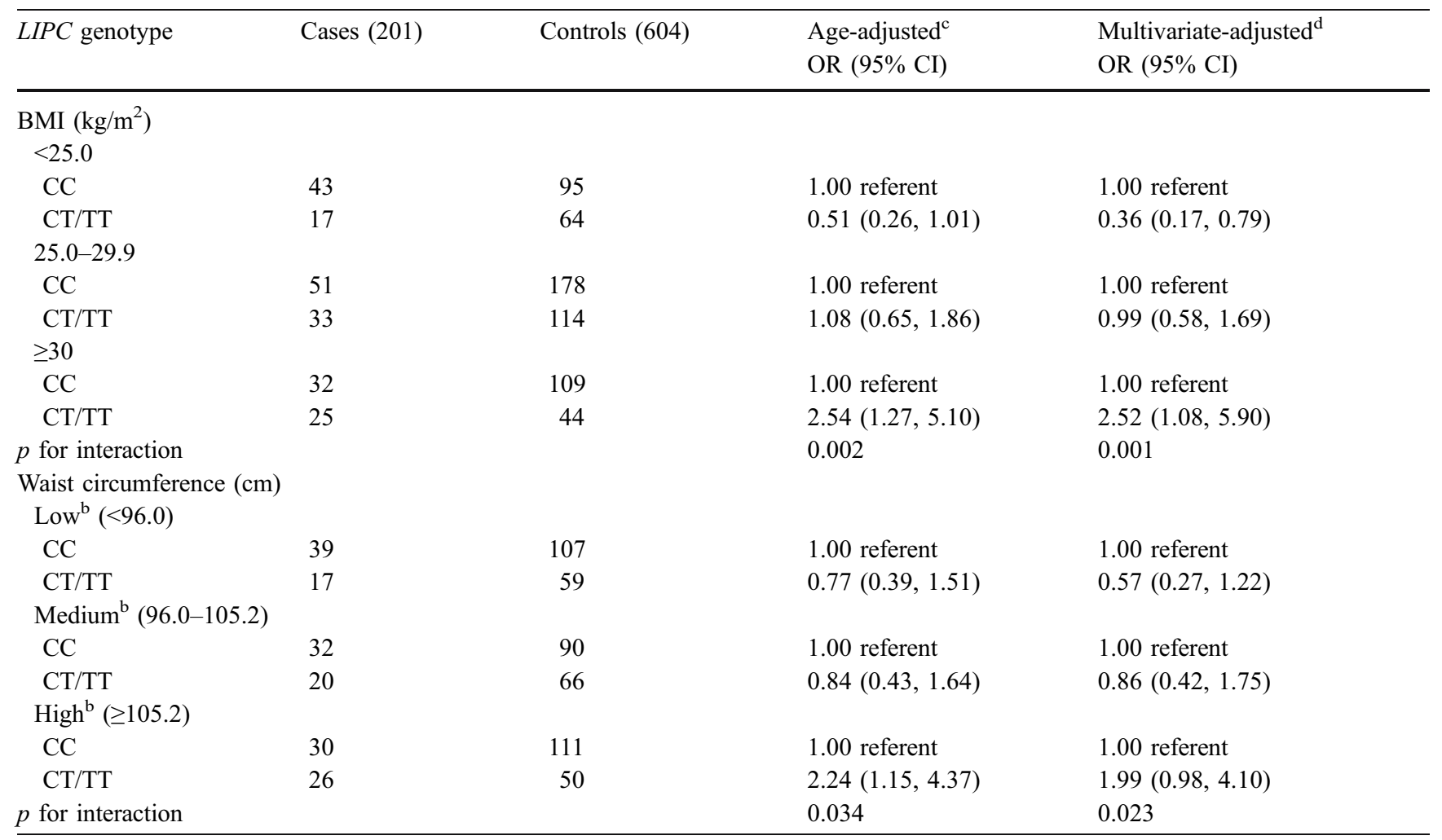

${ }^{a}$ Measurements of waist circumference were available for 164 cases and 483 controls

${ }^{\mathrm{b}}$ Tertile

${ }^{c}$ Adjusted for age $(<55 / 55,59 / 60,64 / 65, \geq 69 / 70$ years $)$

${ }^{\mathrm{d}}$ Adjusted for age, duration of diabetes and the following lifestyle factors: alcohol consumption (non-drinker, $<5.0,<10.0,>10.0 \mathrm{~g} / \mathrm{day}$ ), smoking (never/past/current), physical activity (quartile), and total energy (kcal/day) 
and the $L I P C-514$ polymorphism in relation to $\mathrm{CHD}$ risk were observed.

\section{Discussion}

In this study of US diabetic men, the association between the LIPC C $(-514) \mathrm{T}$ polymorphism and CHD risk was modulated by both overall adiposity and central obesity. Among obese men, the CT or TT genotype was associated with significantly increased risk of CHD compared with those with the CC genotype. However, among lean men this polymorphism was associated with a reduction in CHD risk.

Previous data on the relationship between the LIPC $\mathrm{C}(-514) \mathrm{T}$ polymorphism and CHD have been inconsistent. While several studies suggested a pro-atherogenic role of this polymorphism [24, 26-31], others reported no association or even an inverse association with CHD risk [20, 32-34]. For instance, in a Danish cohort, participants with the TT genotype were observed to have a 1.5 -fold $(95 \% \mathrm{CI}$ $1.0,2.2)$ increased risk of ischaemic heart disease compared with those with the CC genotype [29]. The extent of coronary atherosclerosis determined by angiography was reported to be higher in participants with the T allele [27]. The $L I P C-514 \mathrm{~T}$ allele was also reported to be associated with a decrease in coronary flow reserve [28] and the presence of subclinical CHD [26]. In contrast, no association of the LIPC C $(-514) \mathrm{T}$ polymorphism with the risk for CHD or coronary artery disease was observed in a study of Finnish men [20]. In another cohort of Finnish men, men with the $\mathrm{CC}$ genotype had elevated risk of acute myocardial infarction compared with those having the CT or TT genotype (relative risk=1.5) [34]. Some of the inconsistencies may be due, at least in part, to different study population characteristics, such as underlying environmental and/or physiological characters, which can affect LIPC activity and HDL metabolism. In the present study we found that the association between the LIPC $\mathrm{C}(-514) \mathrm{T}$ polymorphism and CHD risk was dependent on overall adiposity and central obesity status. Previous studies that did not account for these gene-environment interactions may have obscured the effect of this polymorphism on CHD risk.

The precise mechanism by which obesity modifies the association between the LIPC $\mathrm{C}(-514) \mathrm{T}$ polymorphism and CHD risk has yet to be elucidated. The LIPC -514 polymorphism is a key determinant of LIPC activity. The T allele has been shown to decrease transcriptional activity of the $L I P C$ promoter in vitro $[21,22]$ and to account for up to $38 \%$ of the variation in LIPC activity [23]. LIPC activity is also affected by body adiposity; both BMI [10] and intraabdominal fat [45] were strongly and positively associated with LIPC activity. We speculated that the observed interaction between the LIPC polymorphism and adiposity in association with CHD risk may act through their influence on LIPC activity and be related to the dual roles of LIPC activity in lipoprotein metabolism. LIPC activity has both pro-atherogenic and anti-atherogenetic properties
[4]. LIPC catalyses the conversion of large HDL2 particles to small dense HDL by modulating the triglyceride and phospholipids content of these particles. It can also act on large LDL and IDL particles to form small dense LDL particles [3-5]. On the other hand, as a ligand, LIPC can be potentially anti-atherogenic through stimulating reverse cholesterol transport and clearing IDL [4]. The LIPC $\mathrm{C}(-514) \mathrm{T}$ polymorphism associated with lower hepatic lipase activity may, therefore, have both anti-atherogenic and pro-atherogenetic effects. Which effect prevails may be dependent on other factors. It is possible that, in the presence of obesity-related metabolic changes among diabetic patients the pro-atherogenic property related to the LIPC polymorphism outweighs the anti-atherogenic property, which may consequently lead to an elevated risk of CHD. Conversely, among lean diabetic men, the antiatherogenic effect may prevail. The relationship among the LIPC -514 polymorphism, adiposity and CHD could be complicated by pathophysiological and metabolic changes associated with diabetes. We are unaware of experimental data on the relationship among diabetic patients.

In agreement with the findings from the present study, an interaction of similar direction between the LIPC polymorphism and adiposity in association with HDL-C concentrations was observed in some studies $[35,46]$. In a study of French-Canadian men [46], only lean carriers of the $\mathrm{T}$ allele had higher plasma HDL-C levels compared with lean $\mathrm{CC}$ homozygotes. The beneficial effect of the $\mathrm{T}$ allele was abolished in the presence of visceral obesity. Similarly, among 780 US diabetic men who were free of cardiovascular diseases at the time of drawing blood (the source cohort of the controls in the present study), we observed that overall adiposity significantly modified the association between the LIPC polymorphism and plasma HDL-C concentrations [35]. Only among lean men (BMI $<25 \mathrm{~kg} / \mathrm{m}^{2}$ ) was the T allele associated with significantly elevated plasma HDL-C levels; there was no association in overweight or obese men. In the present study, the interaction between adiposity and the LIPC polymorphism was attenuated but remained significant after controlling for plasma HDL concentrations, which indicated that the observed interaction was due in part, but not entirely, to a differential effect of the $L I P C-514$ polymorphism on HDL concentrations by obesity status. Experimental studies with measurements of the LIPC polymorphism, LIPC activity and lipoprotein profiles are warranted to explore the physiological and functional significance of this polymorphism for atherosclerosis and CHD risk in the context of obesity.

One limitation of our study is that the HPFS does not represent a random sample of US diabetic men. The variant allele frequency (0.20) in this study, however, is in agreement with that reported in previous studies among people of European extraction [24, 47]. Secondly, we cannot exclude the possibility that the observed association with CHD is due to linkage disequilibrium with other, undiscovered variants in this gene or to its interaction with other genes important in determining plasma HDL-C levels or $\mathrm{CHD}$ risk; for example, the apolipoprotein $\mathrm{AI} / \mathrm{CIII} / \mathrm{AIV}$, 
cholesteryl ester transfer protein and lipoprotein lipase loci. Thirdly, we cannot rule out the possibility that the observed significant interaction may be due to residual confounding by unmeasured factors. We controlled for major measured risk factors for CHD and the findings did not change appreciably. Lastly, we cannot completely exclude the possibility that our results may be due to chance. It should be noted, however, that we conducted the present study based on our a priori finding of significant interactions between the LIPC -514 polymorphism and adiposity in determining plasma HDL concentrations [35]. Nonetheless, it will be important to confirm these findings with additional investigations among large samples of diabetic patients.

In conclusion, we observed heterogeneity of the association of the LIPC C(-514)T polymorphism with CHD risk according to obesity status among diabetic men. The increase in CHD risk associated with the polymorphism was observed only for obese men. These data support a role for gene-environment interaction in susceptibility to CHD among diabetic patients and, if confirmed, may provide an additional impetus for reducing obesity to prevent CHD.

Acknowledgement This research was supported by awards from the National Institutes of Health (HL 65582 and HL 35464).

\section{References}

1. Garg A, Grundy SM (1990) Management of dyslipidemia in NIDDM. Diabetes Care 13:153-169

2. Cohen JC, Wang Z, Grundy SM, Stoesz MR, Guerra R (1994) Variation at the hepatic lipase and apolipoprotein AI/CIII/AIV loci is a major cause of genetically determined variation in plasma HDL cholesterol levels. J Clin Invest 94:2377-2384

3. Deeb SS, Zambon A, Carr MC, Ayyobi AF, Brunzell JD (2003) Hepatic lipase and dyslipidemia: interactions among genetic variants, obesity, gender, and diet. J Lipid Res 44:1279-1286

4. Jansen H, Verhoeven AJ, Sijbrands EJ (2002) Hepatic lipase: a pro- or anti-atherogenic protein? J Lipid Res 43:1352-1362

5. Applebaum-Bowden D (1995) Lipases and lecithin: cholesterol acyltransferase in the control of lipoprotein metabolism. Curr Opin Lipidol 6:130-135

6. Thuren T (2000) Hepatic lipase and HDL metabolism. Curr Opin Lipidol 11:277-283

7. Cooper AD (1997) Hepatic uptake of chylomicron remnants. J Lipid Res 38:2173-2192

8. Tan CE, Foster L, Caslake MJ et al (1995) Relations between plasma lipids and postheparin plasma lipases and VLDL and LDL subfraction patterns in normolipemic men and women. Arterioscler Thromb Vasc Biol 15:1839-1848

9. Carr MC, Hokanson JE, Zambon A et al (2001) The contribution of intraabdominal fat to gender differences in hepatic lipase activity and low/high density lipoprotein heterogeneity. J Clin Endocrinol Metab 86:2831-2837

10. Nie L, Wang J, Clark LT et al (1998) Body mass index and hepatic lipase gene (LIPC) polymorphism jointly influence ostheparin plasma hepatic lipase activity. J Lipid Res 39: $1127-1130$

11. Dreon DM, Fernstrom HA, Campos H, Blanche P, Williams PT, Krauss RM (1998) Change in dietary saturated fat intake is correlated with change in mass of large low-density-lipoprotein particles in men. Am J Clin Nutr 67:828-836
12. Campos H, Dreon DM, Krauss RM (1995) Associations of hepatic and lipoprotein lipase activities with changes in dietary composition and low density lipoprotein subclasses. J Lipid Res 36:462-472

13. Taskinen MR, Valimaki M, Nikkila EA, Kuusi T, Ylikahri R (1985) Sequence of alcohol-induced initial changes in plasma lipoproteins (VLDL and HDL) and lipolytic enzymes in humans. Metabolism 34:112-119

14. Valimaki M, Kahri J, Laitinen K et al (1993) High density lipoprotein subfractions, apolipoprotein A-I containing lipoproteins, lipoprotein (a), and cholesterol ester transfer protein activity in alcoholic women before and after ethanol withdrawal. Eur J Clin Invest 23:406-417

15. Giada F, Baldo-Enzi G, Baiocchi MR et al (1991) Specialized physical training programs: effects on serum lipoprotein cholesterol, apoproteins A-I and B and lipolytic enzyme activities. J Sports Med Phys Fitness 31:196-203

16. Mendoza SG, Carrasco H, Zerpa A et al (1991) Effect of physical training on lipids, lipoproteins, apolipoproteins, lipases, and endogenous sex hormones in men with premature myocardial infarction. Metabolism 40:368-377

17. Teran-Garcia M, Santoro N, Rankinen T et al (2005) HERITAGE Family Study. Hepatic lipase gene variant $-514 \mathrm{C}>\mathrm{T}$ is associated with lipoprotein and insulin sensitivity response to regular exercise: the HERITAGE Family Study. Diabetes 54:2251-2255

18. Cohen JC, Vega GL, Grundy SM (1999) Hepatic lipase: new insights from genetic and metabolic studies. Curr Opin Lipidol 10:259-267

19. Guerra R, Wang J, Grundy SM, Cohen JC (1997) A hepatic lipase (LIPC) allele associated with high plasma concentrations of high density lipoprotein cholesterol. Proc Natl Acad Sci USA 94:4532-4537

20. Tahvanainen E, Syvanne M, Frick MH et al (1998) Association of variation in hepatic lipase activity with promoter variation in the hepatic lipase gene. The LOCAT Study Investigators. J Clin Invest 101:956-960

21. Deeb SS, Peng R (2000) The C-514T polymorphism in the human hepatic lipase gene promoter diminishes its activity. J Lipid Res 41:155-158

22. Botma GJ, Verhoeven AJ, Jansen H (2001) Hepatic lipase promoter activity is reduced by the C-480T and G-216A substitutions present in the common LIPC gene variant, and is increased by upstream stimulatory factor. Atherosclerosis 154:625-632

23. De Oliveira e Silva ER, Kong M, Han Z et al (1999) Metabolic and genetic determinants of HDL metabolism and hepatic lipase activity in normolipidemic females. J Lipid Res 40:1211-1221

24. Jansen H, Verhoeven AJ, Weeks L et al (1997) Common C-to-T substitution at position -480 of the hepatic lipase promoter associated with a lowered lipase activity in coronary artery disease patients. Arterioscler Thromb Vasc Biol 17:2837-2842

25. Zambon A, Deeb SS, Hokanson JE, Brown BG, Brunzell JD (1998) Common variants in the promoter of the hepatic lipase gene are associated with lower levels of hepatic lipase activity, buoyant LDL, and higher HDL2 cholesterol. Arterioscler Thromb Vasc Biol 18:1723-1729

26. Hokanson JE, Cheng S, Snell-Bergeon JK et al (2002) A common promoter polymorphism in the hepatic lipase gene (LIPC-480C $>\mathrm{T}$ ) is associated with an increase in coronary calcification in type 1 diabetes. Diabetes 51:1208-1213

27. Dugi KA, Brandauer K, Schmidt N et al (2001) Low hepatic lipase activity is a novel risk factor for coronary artery disease. Circulation 104:3057-3062

28. Fan Y, Laaksonen R, Janatuinen T et al (2001) Hepatic lipase gene variation is related to coronary reactivity in healthy young men. Eur J Clin Invest 31:574-580 
29. Andersen RV, Wittrup HH, Tybjaerg-Hansen A, Steffensen R, Schnohr P, Nordestgaard BG (2003) Hepatic lipase mutations, elevated high-density lipoprotein cholesterol, and increased risk of ischemic heart disease: the Copenhagen City Heart Study. J Am Coll Cardiol 41:1972-1982

30. Hokanson JE, Kamboh MI, Scarboro S, Eckel RH, Hamman RF (2003) Effects of the hepatic lipase gene and physical activity on coronary heart disease risk. Am J Epidemiol 158:836-843

31. Ji J, Herbison CE, Mamotte CD, Burke V, Taylor RR, van Bockxmeer FM (2002) Hepatic lipase gene $-514 \mathrm{C} / \mathrm{T}$ polymorphism and premature coronary heart disease. J Cardiovasc Risk 9:105-113

32. Whiting BM, Anderson JL, Muhlestein JB et al (2005) Intermountain Heart Collaborative Study Group. Candidate gene susceptibility variants predict intermediate end points but not angiographic coronary artery disease. Am Heart J 150:243-250

33. Burdon KP, Langefeld CD, Beck SR et al (2005) Association of genes of lipid metabolism with measures of subclinical cardiovascular disease in the Diabetes Heart Study. J Med Genet 42:720-724

34. Fan YM, Salonen JT, Koivu TA et al (2004) Hepatic lipase C480T polymorphism modifies the effect of HDL cholesterol on the risk of acute myocardial infarction in men: a prospective population based study. J Med Genet 41:e28

35. Zhang C, Lopez-Ridaura R, Rimm EB, Rifai N, Hunter DJ, Hu FB (2005) Interactions between the -514 C-T polymorphism of the hepatic lipase gene and lifestyle factors in relation to high density-lipoprotein concentrations among US diabetic men. Am J Clin Nutr 81:1429-1435

36. National Diabetes Data Group (1979) Classification and diagnosis of diabetes mellitus and other categories of glucose intolerance. Diabetes 28:1039-1057

37. Expert Committee on the diagnosis and classification of diabetes mellitus (1997) Report of the Expert Committee on the diagnosis and classification of diabetes mellitus. Diabetes Care 20:1183-1197
38. Hu FB, Leitzmann MF, Stampfer MJ, Colditz GA, Willett WC, Rimm EB (2001) Physical activity and television watching in relation to risk for type 2 diabetes mellitus in men. Arch Intern Med 161:1542-1548

39. Rose G, Blackburn H (1982) WHO Monograph Series: Cardiovascular Survey Methods. World Health Organization, Geneva

40. Rimm EB, Giovannucci EL, Willett WC et al (1991) Prospective study of alcohol consumption and risk of coronary disease in men. Lancet 338:464-468

41. Stampfer MJ, Willett WC, Speizer FE et al (1984) Test of the National Death Index. Am J Epidemiol 119:837-839

42. Rimm EB, Stampfer MJ, Colditz GA, Chute CG, Litin LB, Willett WC (1990) Validity of self-reported waist and hip circumferences in men and women. Epidemiology 1:466-473

43. Schulze MB, Rimm EB, Li T, Rifai N, Stampfer MJ, Hu FB (2004) C-reactive protein and incident cardiovascular events among men with diabetes. Diabetes Care 27:889-894

44. WHO Expert Committee (1995) Physical status: the use and interpretation of anthropometry. WHO Technical Report Series no. 854. World Health Organization, Geneva

45. Carr MC, Hokanson JE, Deeb SS, Purnell JQ, Mitchell ES, Brunzell JD (1999) A hepatic lipase gene promoter polymorphism attenuates the increase in hepatic lipase activity with increasing intra-abdominal fat in women. Arterioscler Thromb Vasc Biol 19:2701-2707

46. St-Pierre J, Miller-Felix I, Paradis ME et al (2003) Visceral obesity attenuates the effect of the hepatic lipase $-514 \mathrm{C}>\mathrm{T}$ polymorphism on plasma HDL-cholesterol levels in FrenchCanadian men. Mol Genet Metab 78:31-36

47. Ordovas JM, Corella D, Demissie S et al (2002) Dietary fat intake determines the effect of a common polymorphism in the hepatic lipase gene promoter on high-density lipoprotein metabolism: evidence of a strong dose effect in this genenutrient interaction in the Framingham Study. Circulation $106: 2315-2321$ 